Letter to the Editor

\title{
The site of apalutamide in the treatment of prostate cancer
}

\author{
Kajetan Juszczak, Tomasz Drewa
}

'Department of Urology and Andrology, Collegium Medicum, Nicolaus Copernicus University, Bydgoszcz, Poland

2 Department of Urology Memorial Rydygier Hospital, Cracow, Poland

${ }^{3}$ Department of General and Oncological Urology, Nicolaus Copernicus Hospital, Torun, Poland

Correspondence:

Kajetan Juszczak

Department of Urology and Andrology,

Collegium Medicum, Nicolaus

Copernicus University, Bydgoszcz, Poland 85-094 Bydgoszcz, ul. Marii

Skłodowskiej-Curie 9

e-mail:kaj.juszczak@gmail.com

Received:

1.09.2021

Accepted:

22.09.2021

DOI: 10.24292/01.OR.124221921

Copyright $\odot$ Medical Education.

All rights reserved.

\section{ABSTRACT}

Apalutamide belongs to the ARTA group. This drug is a new generation selective androgen receptor inhibitor. Patients with prostate cancer develop resistance to castration testosterone levels over time during hormone therapy. Therefore, it is necessary to consider additional therapeutic options based on drugs from the ARTA group. The article presents the role of apalutamide in the treatment of prostate cancer and its effectiveness. Moreover, attention was drawn to the need for close cooperation between the urologist and the oncologist in order to optimize the treatment of a patient with progressive prostate cancer.

Key words: apalutamide, prostate cancer, nonmetastatic, metastatic, CRPC, CSPC 
A patient with prostate cancer after radical treatment (radical prostatectomy or radiotherapy) may develop the next stages of cancer progression depending on the stage of the disease and histopathological differentiation. The most common sequence of stages of progression includes biochemical relapse, nonmetastatic castration-resistant prostate cancer (nmCRPC), metastatic castration-resistant prostate cancer (mCRPC), and metastatic castration-resistant prostate cancer (mCRPC) in the terminal stage. It should be remembered that prostate cancer is, in most cases, a hormone-sensitive neoplasm, which is used in the case of anti-androgen therapy. Nevertheless, the response to hormone therapy is temporary and the successive stages of progression are observed despite persistent castration levels of testosterone on standard androgen deprivation with the use of GnRH gonadotropin releasing hormone agonists or antagonists.

It is extremely important to accurately diagnose the stage of prostate cancer that is not sensitive to castration testosterone levels and to quickly modify the current therapy. Modern antiandrogens from the ARTA group (androgen receptor targeted agents), e.g. abiraterone, enzalutamide, apalutamide, darolutamide, are used in the treatment of patients with prostate cancer in order to optimize hormone therapy and delay disease progression. Most people after primary radical treatment of prostate cancer (after surgery as well as after radiotherapy) remain under constant urological control. Therefore, the role of the urologist in the optimization of hormonal pharmacotherapy in a patient with progressive prostate cancer seems to be invaluable. With ARTA drugs available in urological armamentarium, it is particularly important in this group of patients who have not yet developed metastases, and the cancer develops resistance to castration testosterone levels (nmCRPC) [1]. A patient with nmCRPC prostate cancer is characterized by a steadily increasing concentration of prostate specific antigen (PSA) despite the use of androgen deprivation, while maintaining castration levels of testosterone, in whom no clinically detectable metastatic disease is found (in imaging studies) [2]. The epidemiological data to date have shown that $10-20 \%$ of prostate cancer patients develop resistance to castration testosterone levels (CRPC stage) within approx 5 years. Over $84 \%$ of patients have metastatic disease at diagnosis of CRPC cancer. In addition, in patients without metastases at diagnosis of cancer at the CRPC stage, 33\% of patients are expected to develop metastases within 2 years of follow-up [3]. The above mentioned facts clearly indicate the need to make a quick decision on the possible modification of standard androgen deprivation, consisting in the combined treatment of androgen deprivation (agonists or antagonists of gonadotropin releasing hormone $\mathrm{GnRH}$ ) with modern antiandrogen drugs from the ARTA group in patients with prostate cancer at the stage of progression, but still at the nmCRPC stage to delay the time to inevitable development of the MCRPC stage.

According to the current guidelines of many scientific societies, ARTA drugs (apalutamide, darolutamide and enzalutamide) should be considered in the treatment of patients with the $\mathrm{nmCRPC}$ stage at high risk of rapid progression (PSA doubling time $<10$ months) in order to prolongation of the time to metastasis and overall survival (OS) [1].

It is worth noting that the results of a multicentre, double-blind clinical study (phase III) in which 1207 patients with nmCRPC prostate cancer (with PSADT less than 10 months of age) were randomized ( $2: 1)$ to receive apalutamide $240 \mathrm{mg}$ orally once daily in combination with pharmacological castration or surgical (androgen deprivation therapy [ADT) $[n=806]$ or placebo once daily with ADT [ $n=401]$ ) showed the efficacy of apalutamide in this group of patients. In patients treated with apalutamide, an increase in metastasis-free survival (MFS) by 24.3 months was observed [4]. The median MFS was 40.5 months in the apalutamide group and 16.2 months in the placebo group (hazard ratio [HR] $=0.29 ; 95 \%$ confidence interval $[\mathrm{Cl}] 0.24-0.36 ; \mathrm{p}<0.001$ ) [4]. The prolongation of the MFS period resulted in the prolongation of overall survival in the group of nmCRPC prostate cancer patients. In addition, in the apalutamide group, the median OS increased by 14 months compared to the placebo group (73.9 months vs 59.9 months $-\mathrm{HR}=0.78$ [95\% Cl 0.64-0.96]; $\mathrm{p}=0.016)]$ [4]. The median follow-up in the SPARTAN study was 50.4 months. The median duration of treatment with apalutamide and placebo was 32.9 and 11.5 months, respectively [4]. In addition, it is worth noting that apalutamide therapy reduces the risk of initiating cytotoxic chemotherapy by $37 \%$ compared to the placebo group. $\mathrm{HR}=0.63(95 \% \mathrm{Cl} 0.49-0.81) ; \mathrm{p}=0.0002$ (the median was not reached in either group) [4]. The clinical observations to date indicate that the use of a combination therapy (ADT + apalutamide) in a patient with prostate cancer at the nmCRPC stage at high risk of progression first, followed by approved therapies in the mCRPC stage, e.g. abiraterone, enzalutamide, docetaxel, etc., leads to a significantly longer responses than the use of self-treatment for ADT in the nmCRPC stage, followed by approved therapy in the $\mathrm{mCRPC}$ stage $[4,5]$. Thanks to this, we give the patient a chance for another, additional line of treatment. In the case of modern antiandrogen drugs from the ARTA group, pharmacological treatment of a patient with prostate cancer can be carried out at home, taking into account the ease of use (oral treatment) and relatively good tolerability of the therapy. 
It is worth noting that in order to ensure the most effective treatment of people with prostate cancer in the stage of cancer progression, mutual cooperation between the urologist and oncologist is invaluable. Without such close cooperation, achieving a measurable patient clinical response to therapy may be questionable, and achieving the goals of delaying progression, maximizing the time to metastasis and overall survival cannot be achieved. Therefore, the urologist during the oncological supervision of a prostate cancer patient with a biochemical recurrence found during ADT therapy must be alert to the failure to overlook the onset of nmCRPC stage development and the possible need to modify pharmacotherapy based on modern antiandrogen drugs from the ARTA group. Mutual, close cooperation between a urologist who diagnoses non-metastatic nmCRPC prostate cancer and an oncologist who runs drug programs (ARTA drugs) may bring measurable benefits for the patient by slowing down the progression of the disease.

\section{References}

1. Mottet N, Cornford P, van den Bergh RCN et al. (2021). EAU-ESTRO-ESUR-SIOG Guidelines on Prostate Cancer. http://uroweb.org/guideline/ prostate-cancer (access: 8.09.2021).

2. Hong JH, Kim IY. Nonmetastatic castration-resistant prostate cancer. Korean J Urol. 2014; 55(3): 153-60.

3. Kirby M, Hirst C, Crawford ED. Characterising the castration-resistant prostate cancer population: a systematic review. Int J Clin Pract. 2011; 65(11): 1180-92.

4. Smith MR, Saad F, Chowdhury S et al. Apalutamide and Overall Survival in Prostate Cancer. Eur Urol. 2021; 79(1): 150-8.

5. Smith MR, Saad F, Chowdhury S et al; SPARTAN Investigators. Apalutamide Treatment and Metastasis-free Survival in Prostate Cancer. N Engl J Med. 2018; 378(15): 1408-18.

Authors' contributions: Kajetan Juszczak: 50\%; Tomasz Drewa: 50\%

Conflict of interests:

$$
\text { None. }
$$

Financial support:

This research has not received any specific grant from public funding agencies, commercial or non-profit.

Ethics:

The authors had full access to the data and take full responsibility for its integrity.

All authors have read and agreed with the content of the manuscript as written

The paper complies with the Helsinki Declaration, EU Directives and harmonized requirements for biomedical journals. 